\title{
On a Positive Knot without Positive Minimal Diagrams
}

\author{
Takuji NAKAMURA \\ Graduate School of Science and Technology, Kobe University, Rokkoudai 1-1, Nada-ku, Kobe 657-8501, Japan \\ E-mail: tnakamur@math.kobe-u.ac.jp
}

Received June 8, 2002; final version accepted August 6, 2002

\begin{abstract}
A. Stoimenow found an example of a positive knot which has no positive diagram with minimal crossing for the knot. In this note, we give another example inspired by Stoimenow's knot.
\end{abstract}

KEYWORDS: positive knot, minimal diagram, Jones polynomial, HOMFLY polynomial

\section{Introduction}

A link is a closed oriented 1-manifold embedded smoothlyin the 3 -sphere $S^{3}$, and a knot is a link with one connectedcomponent. A link diagram (or diagram) is a generic projection of a link in $S^{3}$ onto the 2-sphere $S^{2} \subset S^{3}$, which contains the information such that the overcrossing line is marked for each doublepoint so that the original knot can be reconstructed. Each crossingin a link diagram has a sign, namely, positive (1) or negative ( A knot is said to be positive if it has a diagram whose all crossings are positive. We call such a diagram a positive diagram. Positive knots have many interesting questions to provide us in analogy to alternating knots. An alternating knot is defined as a knot which has an alternating diagram. A diagram is said to be alternating if an overcrossing and an undercrossing appear alternately as one goes along each component. The minimal crossing number of a knot is the minimal number of crossings that occur in any diagram presenting the knot. We call a diagram realizing the minimal crossing number of a knot a minimal diagram. It is quite hard to determine the minimal crossing number of a given knot, to determine whether a given diagram is minimal or not. We have known the following famous theorem concerning a minimal diagram proved by Kauffman, Murasugi and Thistlethwaite:

Theorem $1.1([\mathbf{5}, \mathbf{1 0}, \mathbf{2 3}])$. Any reduced alternating diagram is a minimal diagram. Moreover, any minimal diagram of a prime alternating knot is alternating.

A diagram is said to be reduced if there exists no crossing such that the diagram is separated by splicing the crossing.

We consider Theorem 1.1 for the case of positive knots. There exists, however, a positive knot with a reduced positive diagram which is not minimal. An example of such a knot is the trefoil knot. See Fig. 1.

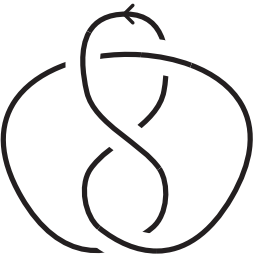

(a)

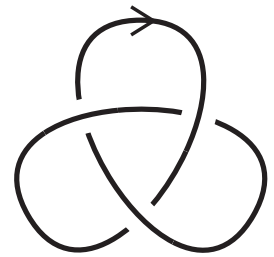

(b)

Fig. 1.

The latter claim of Theorem 1.1 also no longer holds for the case of positive knots, that is, there exists a knot with a positive diagram and a non-positive diagram as a minimal diagram though the positive knot is prime. An example of such a knot is a famous knot called Perko's pair $10_{161}$ and $10_{162}$ in the knot table in Rolfsen's book [19]. The knots $10_{161}$ and $10_{162}$ had been considered as different knots for a long time since knots had been tabulated by Little or Tait in the end of 19th century. In 1970, Perko [18] pointed out that they were equivalent to each other. The diagram of Fig. 2(a) presenting the knot $10_{161}$ is a non-positive diagram. Only the crossing in the dotted circle is negative. The diagram of Fig. 2(b) presenting the knot $10_{162}$ is a positive diagram.

So the question whose affirmative answer corresponds to Theorem 1.1 is the following: 


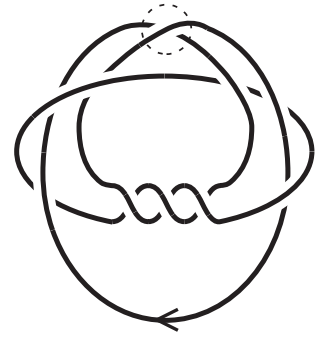

(a)

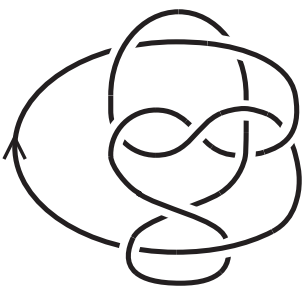

(b)

Fig. 2.

Question 1.2. Is a minimal diagram of any positive knot realized by a positive diagram?

This question is affirmatively solved for torus knots [12], positive alternating knots [15] and positive knots of genus one or two [20,21].

Throughout this paper, all manifolds are oriented in $S^{3}$ unless otherwise stated. For the definition of standard terms in Knot Theory, we refer to $[1,6,13,19]$.

\section{Stoimenow's Example}

Stoimenow [22] found the following example of a positive knot which gives a negative answer for Question 1.2, illustrated in Fig. 3. We call this knot Stoimenow's knot.

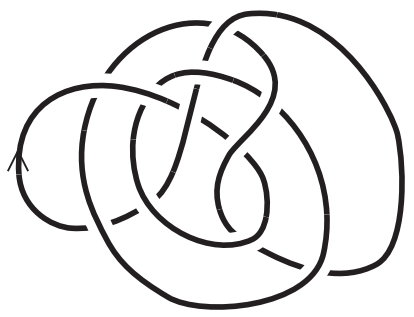

(a)

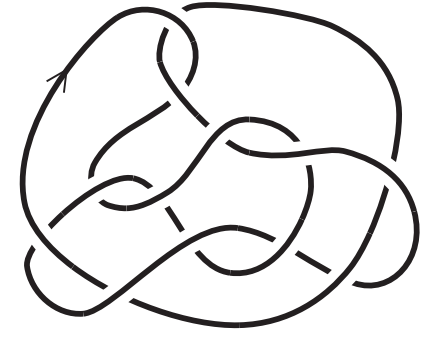

(b)

Fig. 3.

The diagram in Fig. 3(a) is a non-positive diagram with 11 crossings. This knot has a positive diagram with 12 crossings as in Fig. 3(b). (It follows Fig. 4 that these two diagrams present the same knot. The left most diagram in Fig. 4 is obtained from the diagram in Fig. 3(a) by turning it over.)
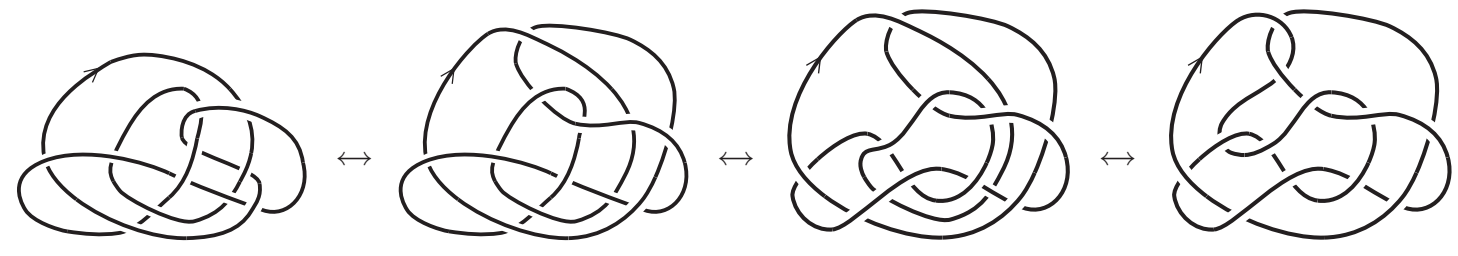

Fig. 4.

By a precise observation of this deformation, we see that the interior of the circle in Fig. 5(a) is not affected by this deformation. So we obtain a tangle where this deformation completes inside. See Fig. 5(b). We will construct a positive knot from this tangle, and show that it has no positive diagram with minimal crossings in the next section. 


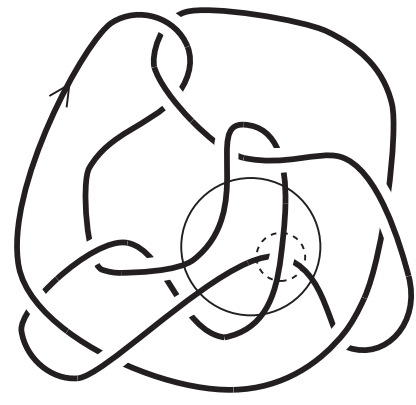

(a)

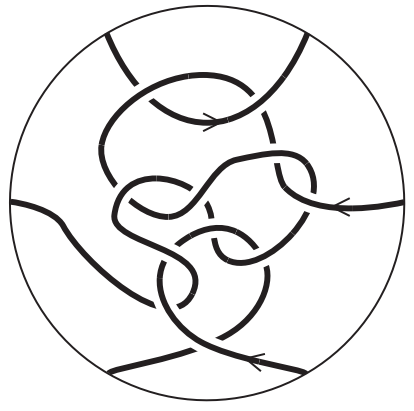

(b)

Fig. 5.

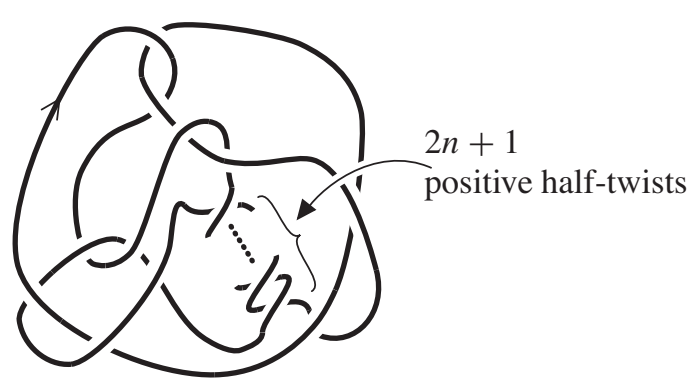

(a)

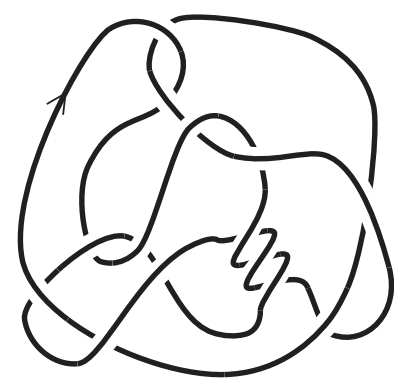

(b)

Fig. 6.

\section{Positive Knot without Positive Minimal Diagrams}

Let $S_{0}$ be Stoimenow's knot. Let $S_{n}$ be the positive knot as in Fig. 6(a), obtained from $S_{0}$ by changing the crossing in the dotted circle in Fig. 5(a) into $2 n+1$ positive half-twists.

Ozawa [17, Theorem 1.4.] showed that if a nontrivial positive knot is prime then its reduced positive diagram is prime. A diagram $D$ on the 2 -sphere $S$ is said to be prime if for any simple loop $l$ on $S$ intersecting $D$ in 2 points, then $l$ bounds a disk intersecting $D$ in a simple arc. Hence we see that the positive knot $S_{n}$ is prime.

In this note, we concentrate on the positive knot $S_{1}$ [Fig. 6(b)]. We note that the positive knot $S_{1}$ has a positive diagram with 14 crossings and its minimal crossing number is 13 or less. Then we have the following:

Theorem 3.1. The positive knot $S_{1}$ has no positive diagram with 13 crossings or less.

Hence the positive knot $S_{1}$ is an example of a positive knot without a positive minimal diagram.

Remark 3.2. If we consider this theorem for a non-prime positive knot, then we can easily obtain a positive knot without a positive minimal diagram by the composition of several copies of Stoimenow's knot $S_{0}$. Let $K$ be the composite knot of $n$ copies of $S_{0}$ and $c^{+}(K)$ the minimal crossing number that occur in any positive diagram presenting $K$. We see that the minimal crossing number $c(K)$ of $K$ is at most $11 n$. Now we can see that $c^{+}(K) \leq 12 n$. However, if $c^{+}(K)<12 n$ then $S_{0}$ must have a positive diagram with 11 crossings or less since any positive diagram of a composite positive knot is non-prime by Ozawa [17, Theorem 1.4.]. This is a contradiction. Thus we have $c^{+}(K)=12 n$. Hence the difference between $c(K)$ and $c^{+}(K)$ can be arbitrarily large.

We propose the following conjecture.

Conjecture 3.3. The positive knot $S_{n}$ has no positive diagram with the minimal crossing for $S_{n}$ for $n \geq 2$.

In order to prove Theorem 3.1 in the simplest way, we first list up the positive diagrams with 13 crossings or less and show that the positive knot $S_{1}$ is not contained in the list by some invariants. It is, however, not effective since there exists a huge number of positive diagrams with 13 crossings or less. We consider some properties of $S_{1}$ in order to reduce the number of positive diagrams to be investigated.

Algebraic properties: Let $V_{K}(t)$ be the Jones polynomial of a knot $K$, which calculated by the following recursive relations. 
(1) $V_{O}(t)=1$,

(2) $t^{-1} V_{K_{+}}(t)-t V_{K_{-}}(t)=\left(t^{-1 / 2}-t^{1 / 2}\right) V_{K_{0}}(t)$,

where $O$ is the trivial knot and $K_{+}, K_{-}$and $K_{0}$ are three links that are identical except near one point $\longrightarrow$ respectively.

By an easy calculation, we have

$$
V_{S_{1}}(t)=t^{15}-3 t^{14}+5 t^{13}-8 t^{12}+9 t^{11}-10 t^{10}+9 t^{9}-6 t^{8}+4 t^{7}-t^{6}+t^{4}
$$

We also have the ordinary knot signature $\sigma\left(S_{1}\right)$ of the positive knot $S_{1}$ is equal to -6 by an easy calculation.

There exists the following theorem concerning the relation between the degree of the Jones polynomial and the number of crossings in a diagram.

Theorem 3.4 ([11]). For any knot $K$ and any diagram $D$ of $K$,

$$
\begin{aligned}
& \max \operatorname{deg} V_{K}(t)-\min \operatorname{deg} V_{K}(t) \leq c(D), \\
& \max \operatorname{deg} V_{K}(t)+\frac{1}{2} \sigma(K) \leq c_{+}(D),
\end{aligned}
$$

where $c(D)\left(\right.$ resp. $\left.c_{+}(D)\right)$ is the number of crossings (resp. positive crossings) in $D$.

Then we have

$$
\begin{aligned}
& 11 \leq c\left(S_{1}\right), \\
& 12 \leq c_{+}(D),
\end{aligned}
$$

where $D$ is a diagram of $S_{1}$. By the inequality (1), it is enough to check the existence of positive diagrams of $S_{1}$ with 13 , 12 and 11 crossings. However, it is unnecessary to check the existence of positive diagrams with 11 crossings by the inequality (2).

Topological properties: A knot is said to be fibered if the complement of the knot is a surface bundle over a circle, whose fiber is a Seifert surface of the knot. It is known that the Seifert surface as a fiber of a fibered knot is unique up to ambient isotopy and gives the minimal genus of the knot (for example, see [8] and [7]). It is known that a Seifert surface obtained from a positive diagram by applying Seifert's algorithm gives the minimal genus of the knot $([2,14])$. We can see that a Seifert surface of the positive knot $S_{1}$ obtained from the positive diagram in Fig. 6(b) is the Hopf plumbing, namely, it is constructed from several Hopf bands by plumbing. The Hopf band is the fiber surface of the Hopf link. Plumbing is a special case of an operation "Murasugi sum". The Murasugi sum is a way to create a new Seifert surface by pasting two Seifert surfaces along a disk (more precisely $2 n$-gon). We call such a disk a summing disk. Gabai [4] showed that the resulting Seifert surface by the Murasugi sum is a fiber surface if and only if the summands are fiber surfaces. Plumbing is pasting two Seifert surfaces along 4-gon. So $S_{1}$ is a fibered knot of genus 4 (we can also see that $S_{n}$ is a fibered knot of genus $n+3$ ).

The spine of a Seifert surface $F$ obtained from a diagram by applying Seifert's algorithm can be regarded as a graph $G$. The vertices of $G$ correspond to the Seifert disks of $F$. The edges of $G$ correspond to the twisted rectangles of $F$, hence to the crossings in the diagram. We call this graph a Seifert graph. We note that a Seifert graph is a planar bipartite graph. We also note that a cut-vertex (i.e., a vertex which divides the graph into several graphs by deleting itself) of a Seifert graph corresponds to a summing disk of a Seifert surface.

Proposition 3.5. Let $F$ be the fiber surface of a fibered positive knot obtained from a positive diagram $D$ and $G$ the Seifert graph from $F$. Then $G$ has no cycle bounding a disk-face with the length greater than 2 when $G$ is embedded in the 2-sphere $S^{2}$.

Proof. We may assume that $D$ is a special diagram, i.e., any Seifert disk of $F$ is unnested. If not so, we can perform a reversing operation of the Murasugi sum at the nested Seifert disks. This preserves the fiberedness of surfaces. We note a special positive diagram is alternating (for example see [15]). We also assume that $D$ is prime. Hence we may assume that $G$ has no cut-vertex. By [9]: A knot or link with a special alternating diagram is fibered if and only if it is the connected sum of torus knots or links of type $(2, n), G$ must consist of 2 vertices and several edges connecting them.

So the cycles are derived from only multiedges. Since $S_{1}$ is a fibered knot of genus 4 and the number of crossings of a positive diagram which we will check its existence in Sects. 4 and 5 is 12 or 13, its Seifert graph consists of 5 (resp. 6) vertices and 12 (resp. 13) edges. All such graphs are illustrated in Fig. 7 as spanning trees. We assign a positive integer to each edge of graphs $G_{1}, \ldots, G_{9}$. If an edge $e$ joining two vertices $v_{1}$ and $v_{2}$ is assigned an integer $n, e$ presents $n$ edges joining $v_{1}$ and $v_{2}$, namely, a multiedge of multiplicity $n$. Hence each integer is greater than 1 and the sum of integers in each graph of $G_{1}, \ldots, G_{3}$ (resp. graphs $G_{4}, \ldots, G_{9}$ ) is equal to 12 (resp. to 13). There exist five types of assignments for graphs $G_{1}, \ldots, G_{3}$ such as $\{6,2,2,2\},\{5,3,2,2\},\{4,4,2,2\},\{4,3,3,2\}$ and $\{3,3,3,3\}$. There exist three 


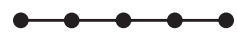

$G_{1}$

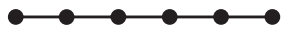

$G_{4}$

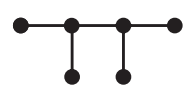

$G_{7}$

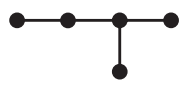

$G_{2}$

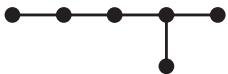

$G_{5}$

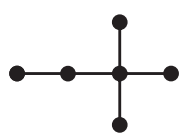

$G_{8}$

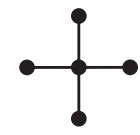

$G_{3}$

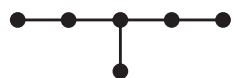

$G_{6}$

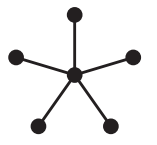

$G_{9}$

Fig. 7.

types of assignments for graphs $G_{4}, \ldots, G_{9}$ such as $\{5,2,2,2,2\},\{4,3,2,2,2\}$ and $\{3,3,3,2,2\}$.

\section{DE-Graph}

For each graph in Fig. 7, we reconstruct a positive diagram and calculate polynomial invariants. However we can reduce the number of graphs to check before calculating polynomial invariants. Let $P_{K}(v, z)=\sum P_{2 i}(v) z^{2 i}$ be the HOMFLY polynomial of a knot, which calculated by the following recursive relations.

(1) $P_{O}(v, z)=1$,

(2) $v^{-1} P_{K_{+}}(v, z)-v P_{K_{-}}(v, z)=z P_{K_{0}}(v, z)$,

where $O, K_{+}, K_{-}$and $K_{0}$ are four links as in the definition of the Jones polynomial. We call a polynomial $P_{2 i}(v)$ the $i$-th coefficient polynomial of $P_{K}(v, z)$.

We can calculate the HOMFLY polynomial for a given link by using only the above recursive relations (1) and (2). Then it is convenient to use a binary tree, called a resolution tree, for calculating the polynomial from a diagram. Let $D$ be a diagram for a link $L$. Choose a crossing $c$ in $D$. For convenience, we assume that $c$ is positive. It is better to rename the diagram $D_{+}$. Then we perform a crossing change and a smoothing at $c$. we denote by $D_{-}$and $D_{0}$ the altered diagrams, respectively. We connect $D_{+}$and $D_{-}$(resp. $D_{+}$and $D_{0}$ ) by drawing a line segment and assign $v^{2}$ (resp. $v z$ ) to the line segment. See Fig. 8. The appropriate assignment follows from the relation (2). We proceed this procedure for $D_{-}$and $D_{0}$ until the subsequent altered diagrams present trivial links, respectively. This completes a construction of a binary tree to calculate $P_{L}(v, z)$. We can now calculate $P_{L}(v, z)$ by summing up the HOMFLY polynomial of the each terminating trivial link (or knot) multiplied by the product of the coefficients (on the line segments) along the (uniquely determined) branch path that begins with the original diagram $D$ and terminates with the diagram of this trivial link (or knot).

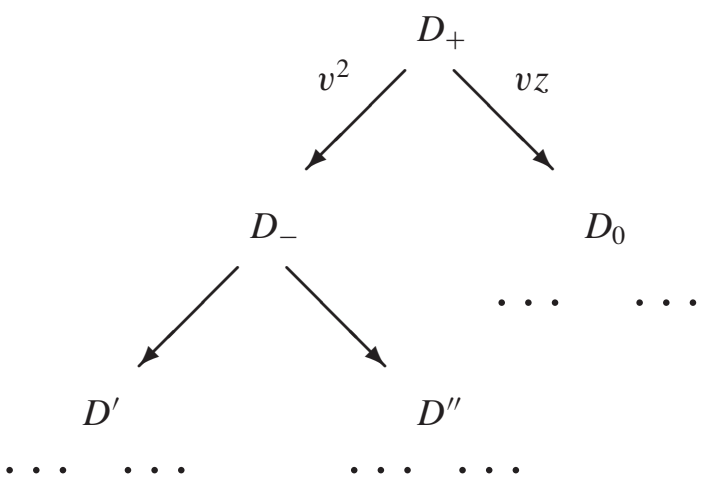

Fig. 8. 
Definition 4.1. Let $\Gamma_{L}$ be a resolution tree to calculate the HOMFLY polynomial for a link $L$. Then $\Gamma_{L}$ is said to be a positive resolution tree if any diagram corresponding to each node is positive and any crossing which will be performed a crossing change or smoothing appears as a positive clasp (i.e., locally presented as a 2-string full-twist $\sigma_{i}^{2}$ for Artin's braid generator $\sigma_{i}$ ). See Fig. 9.

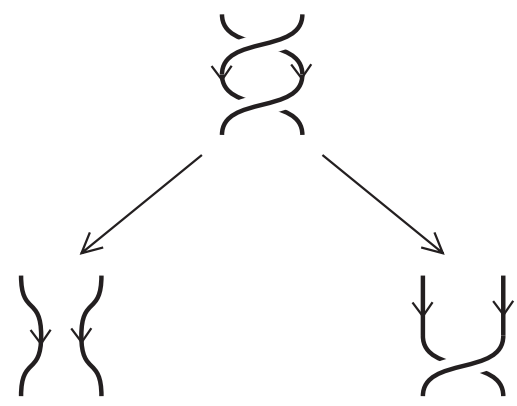

Fig. 9.

Lemma 4.2 ([16, Remark 2.5.]). If a positive knot $K$ has a positive resolution tree, then any coefficient polynomial $P_{2 i}(v)$ is an alternating polynomial.

A polynomial $f(t)=\sum a_{j} t^{j} \in Z\left[t, t^{-1}\right]$ is said to be alternating if $a_{j} a_{j+1}<0$ for any $j$. In this case we say $P_{2 i}(v)=\sum a_{j} v^{2 j}$ is alternating if $a_{j} a_{j+1}<0$ for any $j$. For the positive knots derived from graphs $G_{1}, \ldots, G_{9}$ in Fig. 7, we have the following lemma.

Lemma 4.3. The positive knots derived from the graphs $G_{1}, G_{2}, G_{4}, G_{5}, G_{6}$ and $G_{7}$ in Fig. 7 have positive resolution trees.

Proof. If the maximal number of valency is equal to 2 , namely, graphs $G_{1}$ and $G_{4}$, the positive knots derived from them are closed positive braids. By Franks and Williams [3] or Van Buskirk [24], they have positive resolution trees.

For the other graphs $G_{2}, G_{5}, G_{6}$ and $G_{7}$, we reconsider each vertex as a disk. We call a graph consisting of disks and edges a DE-graph. If the knot diagram constructed by a DE-graph has a positive clasp(two successive edges in a DEgraph) then we can perform a crossing change and a smoothing, and proceed the next stage of a resolution tree. For edges in a DE-graph as in Fig. 10, which illustrates a part of a DE-graph, we can slide an edge. We call this deformation an edge-slide. This deformation corresponds to the Reidemeister move III for a knot diagram. We claim that we can obtain two successive edges by edge-slides or reduce the number of disks.

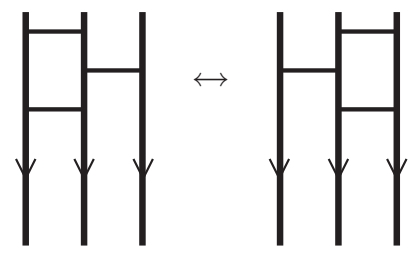

Fig. 10 .

Claim 4.4. Let $D_{1}, \ldots, D_{n}$ be n-tuple disks in a DE-graph. If the partial positive knot diagram derived from the boundaries of the disk $D_{n}$ and parts of disks $D_{1}, \ldots, D_{n-1}$ form an n-braid presented as in Fig. 11, then we obtain one of the following; (1) the disk $D_{n}$ is separated (i.e., $D_{n}$ and $D_{n-1}$ are not connected by an edge), (2) the disk $D_{n}$ can be reduced by edge-slides, (3) there exist two successive edges.

Proof of Claim 4.4. We denote by $e_{i, j}$ the $j$-th edge connecting the $i$-th disk $D_{i}$ and the $(i+1)$-th disk $D_{i+1}$ $\left(1 \leq i \leq n-1,0 \leq j \leq k_{i}\right)$. Let $W$ be the weight of this partial DE-graph defined as $W=\sum_{i=1}^{n-1} i k_{i}$, where $k_{i}$ is the number of edges connecting the $i$-th disk $D_{i}$ and the $(i+1)$-th disk $D_{i+1}$. We assume that the weight $W$ is minimal up to edge-slides.

If there exists no edge $e_{n-1, j}$ then $D_{n}$ is separated. If there exists only one edge $e_{n-1, j}$ then $D_{n}$ is reducible. For both cases Claim 4.4 holds. So we assume that there exist at least two edges $e_{n-1, j}(j \geq 2)$ connecting $D_{n-1}$ and $D_{n}$. 


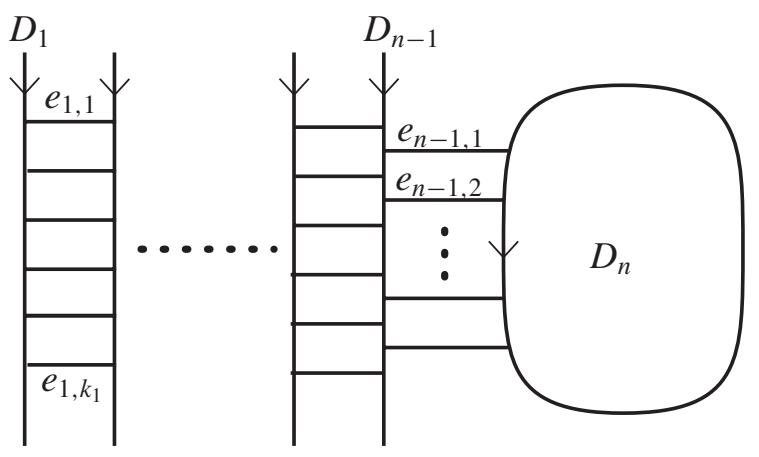

Fig. 11.

If there exists no edge $e_{n-2, j}$ between $e_{n-1,1}$ and $e_{n-1,2}$ then $e_{n-1,1}$ and $e_{n-1,2}$ form two successive edges. If there exists only one edge $e_{n-2, j}$ between $e_{n-1,1}$ and $e_{n-1,2}$ then we can perform an edge-slide and decrease the weight $W$. We have a contradiction to minimality of the weight $W$. If there exist two edges $e_{n-2, j_{1}}$ and $e_{n-2, j_{2}}$ between $e_{n-1,1}$ and $e_{n-1,2}$ then we attend to $e_{n-2, j_{1}}$ and $e_{n-2, j_{2}}$ and proceed the same procedure for them. Finally we attend to edges $e_{2, j_{1}}$ and $e_{2, j_{2}}$. For the cases such that the number of edges between $e_{2, j_{1}}$ and $e_{2, j_{2}}$ is zero or one, we have a conclusion or contradiction by a similar argument for $e_{n-2,1}$ and $e_{n-2,2}$. If there exist several edges $e_{1, j_{1}}, \ldots, e_{1, j_{m}}(m \geq 2)$ between $e_{2, j_{1}}$ and $e_{2, j_{2}}$ then they form two successive edges.

There exist two types of disks of valency 3, such as type 1 or type 2 in Fig. 12.

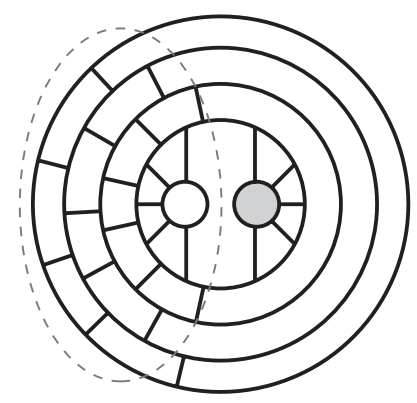

(a) type 1

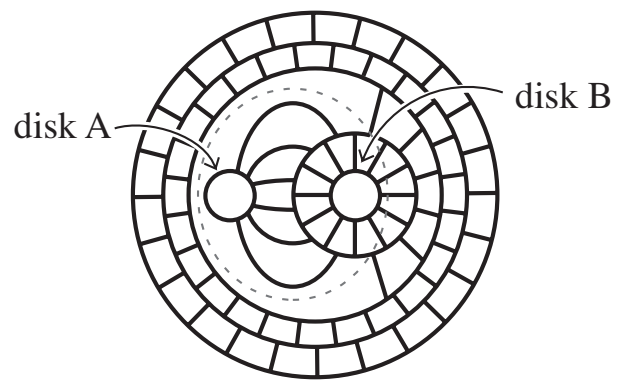

(b) type 2

Fig. 12.

For both types, the number of outer disks can be chosen suitably for each graph $G_{i}(i=2,5,6,7)$. Figure 12 illustrates the case of $G_{5}$.

For type 1. For graphs $G_{2}$ and $G_{5}$, we see that the partial DE-graph in the dotted circle in Fig. 12(a) corresponds to that in Claim 4.4. Hence we can construct a positive resolution tree. For $G_{6}$ and $G_{7}$, we have the same conclusion although there exist disks on the shaded disk in Fig. 12(a).

For type 2. For each graph except $G_{7}$, we can suitably choose the disk corresponding to the disk $D_{n}$ in Claim 4.4 from disks A or B in Fig. 12(b). Then we have the conclusion.

For the graph $G_{7}$, there exists only one case such that we cannot obtain the partial DE-graph corresponding to that in Claim 4.4, that is, we cannot construct a positive resolution tree. The case is the following: There exists the type 1 disk of valency 3 on the disk B in Fig. 12(b). We obtain the DE-graph in Fig. 13 if we construct a DE-graph as few edges as possible so that it realizes the obstruction of the existence of a positive resolution tree. The other cases have positive resolution trees. We put one more edge to somewhere in the DE-graph in Fig. 13 to obtain the DE-graph of $G_{7}$ completely. Then we can see that any resulting DE-graph presents a link diagram. Hence it does not occur. This completes the proof of Lemma 4.3 .

The HOMFLY polynomial of the positive knot $S_{1}$ is the following.

$$
\begin{aligned}
P_{S_{1}}(v, z)= & \left(3 v^{8}+2 v^{10}-6 v^{12}+2 v^{14}\right) \\
& +z^{2}\left(15 v^{8}+3 v^{10}-9 v^{12}+v^{14}\right) \\
& +z^{4}\left(19 v^{8}+3 v^{10}-3 v^{12}\right)
\end{aligned}
$$




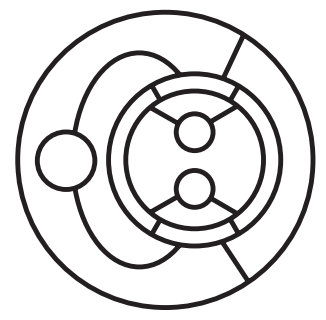

Fig. 13.

$$
\begin{aligned}
& +z^{6}\left(8 v^{8}+v^{10}\right) \\
& +v^{8} z^{8}
\end{aligned}
$$

Since each coefficient polynomial is not alternating, it is enough to check only the positive knot derived from graphs $G_{3}, G_{8}, G_{9}$ by Lemmas 4.2 and 4.3 .

For a disk of valency 4 in a DE-graph, we have the four types as in Fig. 14, which illustrates the case of $G_{8}$.

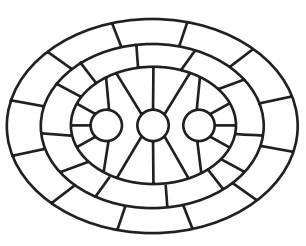

(a)

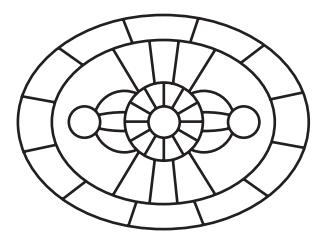

(b)

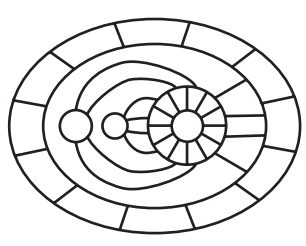

(c)

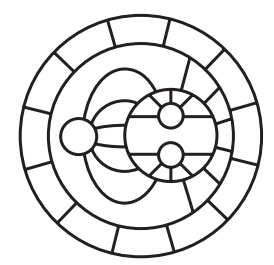

(d)

Fig. 14.

By a similar argument of the proof of Lemma 4.3, we have the following.

Lemma 4.5. The positive knots derived from DE-graphs (a), (b), (c) in Fig. 14 have positive resolution trees.

Hence for DE-graphs with the maximal valency 4 it is enough to check the positive knots derived from DE-graphs of type (d).

\subsection{Graph $G_{3}$}

For checking of the positive knots derived from the graph $G_{3}$, we first construct DE-graphs from the graph $G_{3}$ according to the assignments of positive integers to edges.

For the assignments $\{6,2,2,2\}$ and $\{5,3,2,2\}$ we have DE-graphs in Fig. 15 without 6 or 5 edges corresponding to the edge in $G_{3}$ assigned 6 or 5 .

$\{6,2,2,2\}$

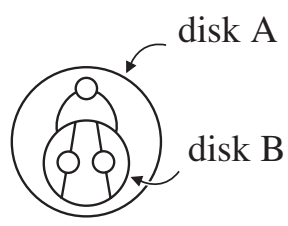

$\{5,3,2,2\}$
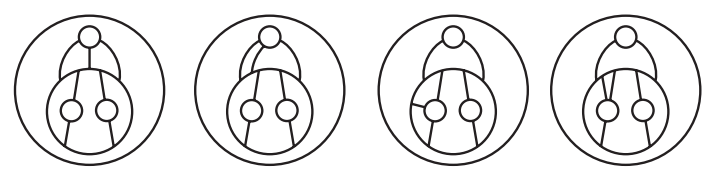

Fig. 15 .

We put 6 or 5 edges between the disk A and the disk B (we also denote these disks by A and B for the other cases) as in Fig. 15 to construct DE-graphs completely. Then we must construct each DE-graph so that it does not present a link diagram or a composite knot diagram, its one of disks is not reduced by edge-slides as in Fig. 16. 


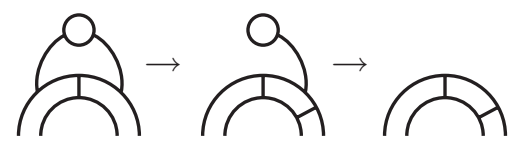

Fig. 16.

Then we obtain 24 DE-graphs.

Next we consider the DE-graphs with the assignment $\{4,4,2,2\}$. For this assignment, we have DE-graphs in Fig. 17.
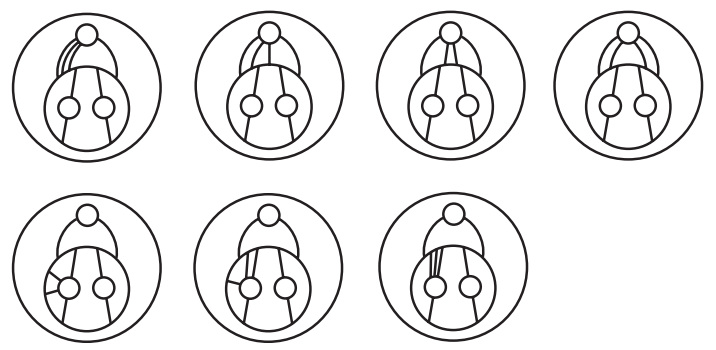

Fig. 17.

We put 4 edges between the disk A and the disk B to construct DE-graphs completely. Then we can see that any resulting DE-graph presents a link diagram or a composite knot diagram, or can be deformed into one of the previous DE-graphs by edge-slides and ambient isotopy. Then we can see that this assignment does not contribute to yielding DE-graphs to be checked.

For the assignment $\{4,3,3,2\}$, we have DE-graphs in Fig. 18.

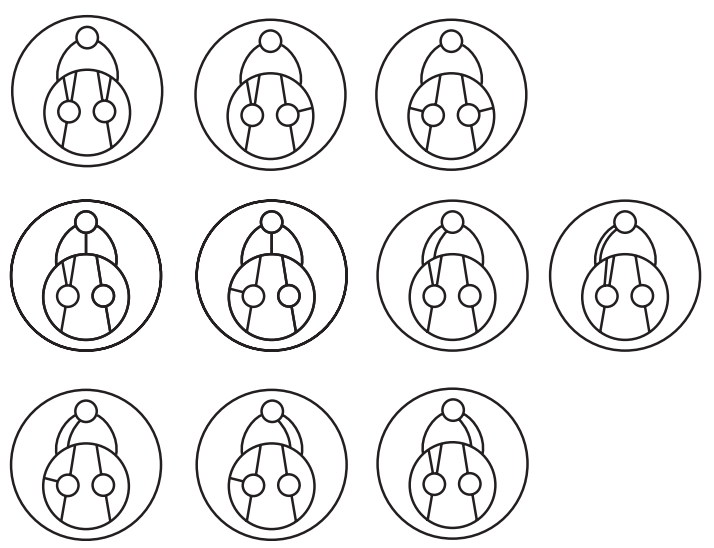

Fig. 18.

Then we obtain 12 DE-graphs by putting 4 edges with the above conditions.

For the assignment $\{3,3,3,3\}$ we have DE-graphs in Fig. 19 without 3 edges corresponding to one of the edges in $G_{3}$ assigned 3.

Then we obtain 11 DE-graphs with the above conditions.

We will check polynomial invariants for the positive knots derived from the above DE-graphs in Sect. 5.

\subsection{Graph $G_{8}$}

The assignment for the edges in the graph $G_{8}$ depends on which integers are assigned to the two edges joined by the vertex of valency 2 , such as $(5,2),(2,5),(2,2)$ in the assignment $\{5,2,2,2,2\},(4,3),(3,4),(4,2),(2,4),(3,2),(2,3)$, $(2,2)$ in the assignment $\{4,3,2,2,2\}$ and $(3,3),(3,2),(2,3),(2,2)$ in the assignment $\{3,3,3,2,2\}$.

Lemma 4.6. If the assignment for the two edges joined by the vertex of valency 2 is $(3,2),(2,3)$ and $(2,2)$ then any $D E$-graph from the graph $G_{8}$ with such assignments is reducible.

Proof. For any assignment in Lemma 4.6 for the two edges joined by the vertex of valency 2, we have a part of a DEgraph as in Fig. 16. Then we can reduce the number of disks by edge-slides as in Fig. 16. 


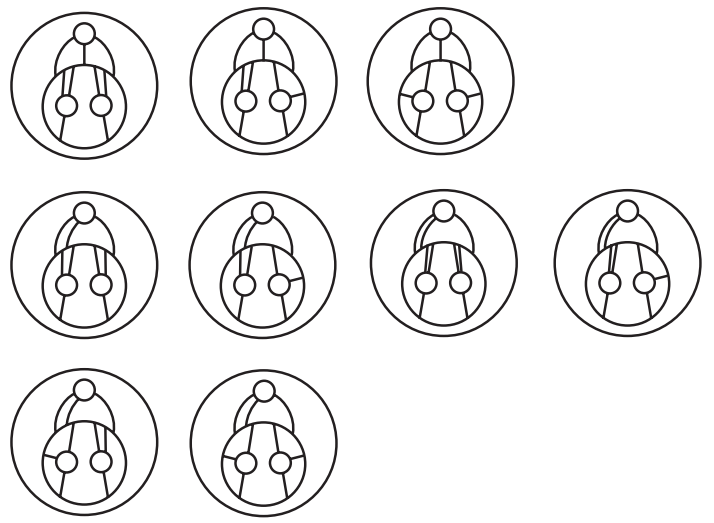

Fig. 19.

We have the following for other assignments, similarly.

Lemma 4.7. If the assignment for the two edges joined by the vertex of valency 2 is $(5,2)$ and $(4,3)($ resp. $(4,2)$ and $(3,3))$ then any DE-graph from the graph $G_{8}$ with such assignments can be deformed into DE-graphs with the assignment $(2,5)$ (resp. $(2,4))$ for the two edges joined by the vertex of valency 2.

Hence it is enough to check only the assignments for the two edges joined by the vertex of valency 2 such as $(2,5)$ in the assignment $\{5,2,2,2,2\},(2,4)$ in the assignment $\{4,3,2,2,2\}$ and $(3,3)$ in the assignment $\{3,3,3,2,2\}$.

We construct DE-graphs from the graph $G_{8}$ with considering conditions mentioned in the construction for $G_{3}$. Then we can see that the graph $G_{8}$ does not contribute to yielding DE-graphs to be checked.

\subsection{Graph $G_{9}$}

For the graph $G_{9}$, we construct DE-graphs by the following way: For each assignment, we first prepare 2 disks and connect them by edges. The number of those edges is the maximal integer in the assignment. Next we put a disk with 2 or 3 edges on the disk of valency 5 corresponding to the vertex of valency 5 in $G_{9}$.

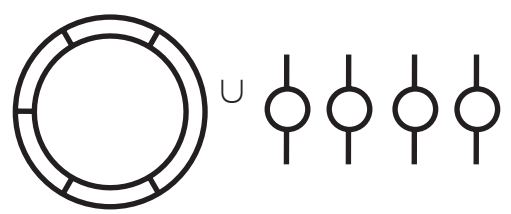

$\{4,3,2,2,2\}$

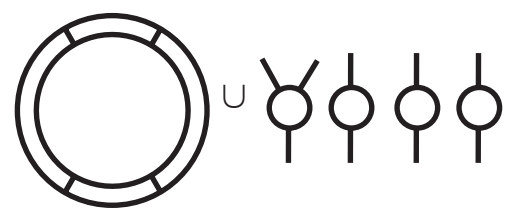

$\{3,3,3,2,2\}$

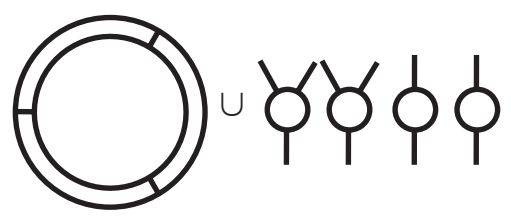

Fig. 20.

Then there exist two ways to put a disk with 2 or 3 edges on the disk of valency 5 , namely, the composition or plumbing. See Fig. 21. (By the primeness of the positive knot $S_{1}$, the composition must be done before the plumbing.)

So we can construct DE-graphs from the graph $G_{9}$ with considering conditions mentioned in the construction for $G_{3}$. We obtain 2 DE-graphs for the assignment $\{5,2,2,2,2\}, 21$ DE-graphs for the assignment $\{4,3,2,2,2\}$ and 71 DEgraphs for the assignment $\{3,3,3,2,2\}$. 


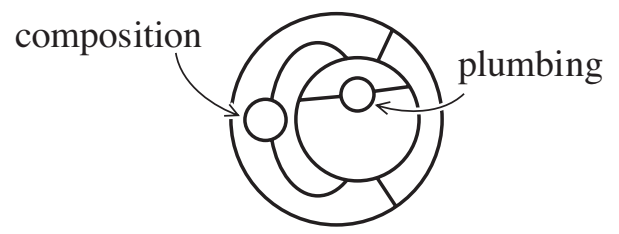

Fig. 21.

\section{Distinction}

In Sect. 4, we obtained 141 DE-graphs (see Appendix) to check their polynomial invariants in order to distinguish the positive knot $S_{1}$ from the positive knots derived from them. We denote by $5_{i}$ (resp. $6_{i}$ ) the $i$-th DE-graph with 5 disks (resp. 6 disks) in the tables in Appendix. There exist, however, positive knots which are mutant to each other among the positive knots derived from them. Two knots are mutant if they can be deformed to each other by flipping a 2-strand tangle (see Fig. 22). It is known that two mutant knots share the same polynomial invariants.

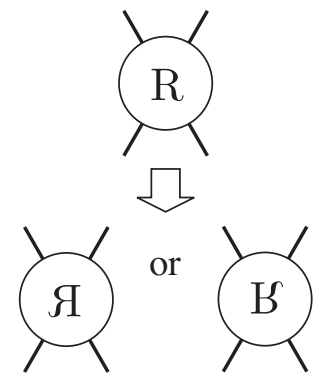

Fig. 22.

Hence we calculate polynomial invariants of the positive knots derived from DE-graphs up to mutant. Then we have 19 positive knots to calculate. For DE-graphs with 5 disks, we have $5_{1}, 5_{2}, 5_{3}, 5_{4}, 5_{5}, 5_{6}, 5_{8}, 5_{10}, 5_{13}, 5_{15}, 5_{23}, 5_{36}$ and $5_{40}$, for DE-graphs with 6 disks, we have $6_{2}, 6_{3}, 6_{4}, 6_{18}, 6_{26}$ and $6_{34}$. The positive knots derived from the other DEgraphs are mutant to them.

So we calculate the Conway polynomial of them. They are in Table 1 .

Table 1. Conway polynomial

\begin{tabular}{||c|c||c|c|}
\hline $5_{1}$ & $1+12 z^{2}+18 z^{4}+8 z^{6}+z^{8}$ & $5_{2}$ & $1+11 z^{2}+17 z^{4}+8 z^{6}+z^{8}$ \\
\hline $5_{3}$ & $1+10 z^{2}+18 z^{4}+8 z^{6}+z^{8}$ & $5_{4}$ & $1+9 z^{2}+16 z^{4}+8 z^{6}+z^{8}$ \\
\hline $5_{5}$ & $1+9 z^{2}+17 z^{4}+8 z^{6}+z^{8}$ & $5_{6}$ & $1+10 z^{2}+17 z^{4}+8 z^{6}+z^{8}$ \\
\hline $5_{8}$ & $1+8 z^{2}+16 z^{4}+8 z^{6}+z^{8}$ & $5_{10}$ & $1+7 z^{2}+14 z^{4}+8 z^{6}+z^{8}$ \\
\hline $5_{13}$ & $1+9 z^{2}+16 z^{4}+8 z^{6}+z^{8}$ & $5_{15}$ & $1+8 z^{2}+17 z^{4}+8 z^{6}+z^{8}$ \\
\hline $5_{23}$ & $1+8 z^{2}+15 z^{4}+8 z^{6}+z^{8}$ & $5_{36}$ & $1+8 z^{2}+14 z^{4}+8 z^{6}+z^{8}$ \\
\hline $5_{40}$ & $1+7 z^{2}+13 z^{4}+7 z^{6}+z^{8}$ & $6_{2}$ & $1+11 z^{2}+20 z^{4}+9 z^{6}+z^{8}$ \\
\hline $6_{3}$ & $1+9 z^{2}+18 z^{4}+9 z^{6}+z^{8}$ & $6_{4}$ & $1+9 z^{2}+17 z^{4}+9 z^{6}+z^{8}$ \\
\hline $6_{18}$ & $1+9 z^{2}+19 z^{4}+9 z^{6}+z^{8}$ & $6_{26}$ & $1+7 z^{2}+14 z^{4}+9 z^{6}+z^{8}$ \\
\hline $6_{34}$ & $1+11 z^{2}+18 z^{4}+9 z^{6}+z^{8}$ & & \\
\hline
\end{tabular}

The positive knot $S_{1}$ has the Conway polynomial

$$
\nabla\left(S_{1}\right)=1+10 z^{2}+19 z^{4}+9 z^{6}+z^{8},
$$

hence $S_{1}$ is a distinct knot from the positive knots derived from DE-graphs constructed in Sect. 4. Thus we can see $S_{1}$ has no positive diagram with 13 crossing or less by the above observations. This completes the proof of Theorem 3.1 . 


\section{REFERENCES}

[1] Burde, G., and Zieschang, H., Knots, Walter de Gruyter \& Co., Berlin (1985).

[2] Cromwell, P. R., "Homogeneous links," J. London Math. Soc., 39: 535-552 (1989).

[3] Franks, J., and Williams, R. F., "Braids and the Jones polynomial," Trans. Am. Math. Soc., 303: 97-108 (1987).

[4] Gabai, D., "Detecting fibred links in $S^{3}$," Comment. Math. Helv., 61: 519-555 (1986).

[5] Kauffman, L. H., "State models and the Jones polynomial," Topology, 26: 395-407 (1987).

[6] Kawauchi, A., A survey of knot theory, Birkhäuser Verlag, Basel, (1996).

[7] Kobayashi, T., "Fibered links and unknotting operations," Osaka J. Math., 26: 699-742 (1989).

[8] Kobayashi, T., "Uniqueness of minimal genus Seifert surfaces for links," Topology Appl., 33: 265-279 (1989).

[9] Murasugi, K., "On alternating knots," Osaka J. Math., 12: 277-303 (1960).

[10] Murasugi, K., "Jones polynomials and classical conjectures in knot theory,” Topology, 26: 187-194 (1987).

[11] Murasugi, K., "On invariants of graphs with applications to knot theory," Trans. Am. Math. Soc., 314: 1-49 (1989).

[12] Murasugi, K., "On the braid index of alternating links," Trans. Am. Math. Soc., 326: 237-260 (1991).

[13] Murasugi, K., Knot theory and its applications, Birkhäuser Boston Inc., Boston, MA (1996).

[14] Nakamura, T., "Four-genus and unknotting number of positive knots and links," Osaka J. Math., 37: 441-451 (2000).

[15] Nakamura, T., "Positive alternating links are positively alternating," J. Knot Theory Ramifications, 9: 107-112 (2000).

[16] Nakamura, T., "Notes on the braid index of closed positive braids," preprint (2002).

[17] Ozawa, M., "Closed incompressible surfaces in the complements of positive knots," Comment. Math. Helv., 77: 235-243 (2002).

[18] Perko, Jr., K. A., “On 10-crossing knots,” Port. Math., 38: 5-9 (1982).

[19] Rolfsen, D., Knots and links, Mathematics Lecture Series, No. 7, Publish or Perish Inc., Berkeley, CA (1976).

[20] Stoimenow, A., "Knots of genus two," preprint (1999).

[21] Stoimenow, A., "Knots of genus one or on the number of alternating knots of given genus," Proc. Am. Math. Soc., 129: 21412156 (2001).

[22] Stoimenow, A., "On the crossing number of positive knots and braids and braid index criteria of Jones and Morton-WilliamsFranks," preprint, available math.GT/0110016 (2001).

[23] Thistlethwaite, M. B., “A spanning tree expansion of the Jones polynomial,” Topology, 26: 297-309 (1987).

[24] Van Buskirk, J. M., "Positive knots have positive Conway polynomials," Knot theory and manifolds (Vancouver, B.C., 1983), Springer, Berlin, 146-159 (1985).

\section{Appendix}

The following is tables of DE-graphs constructed in Sect. 4. (Continued on next page.) 
Table 2. DE-graphs with 5 disks
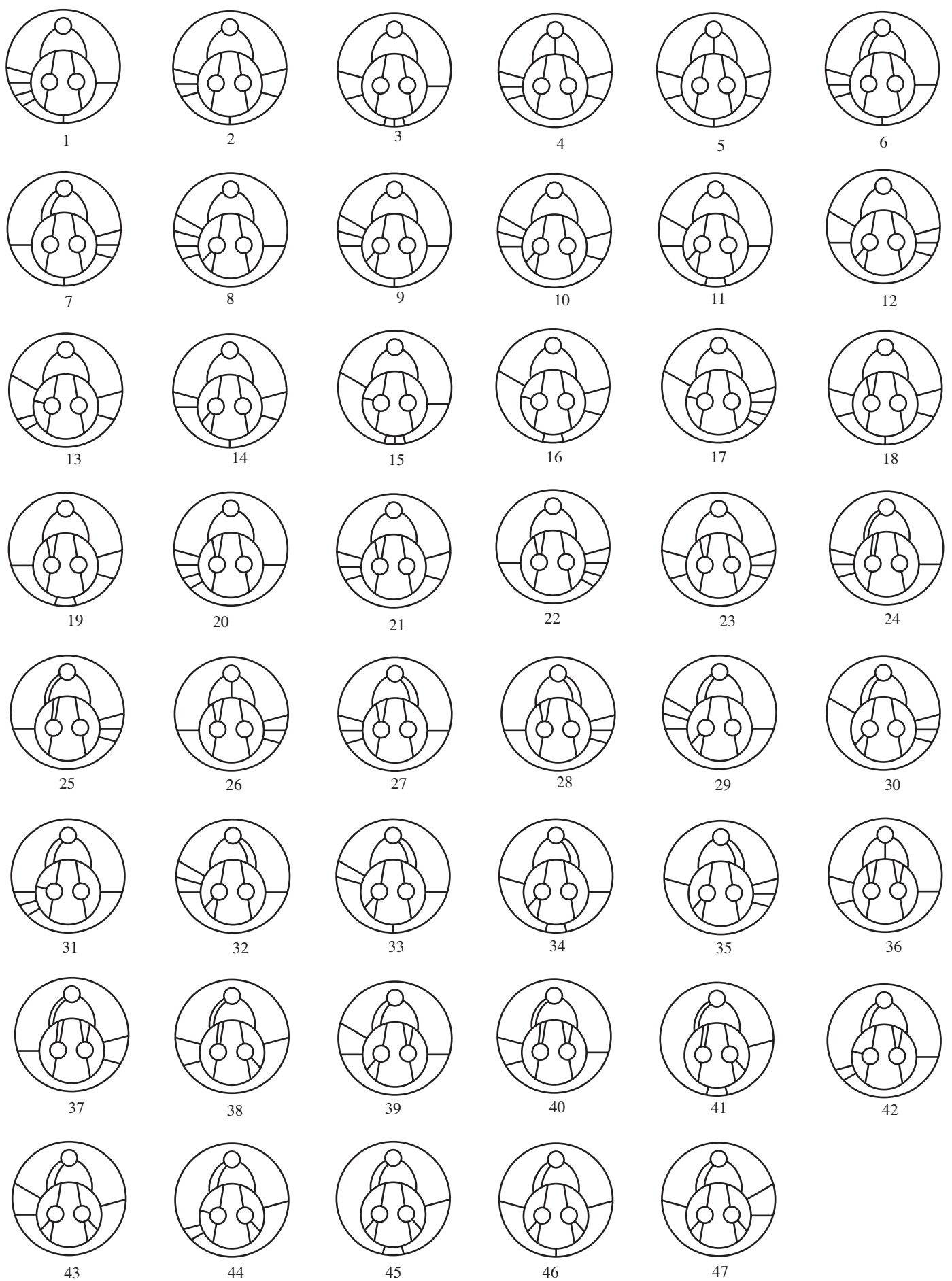
Table 3. DE-graphs with 6 disks

(609)
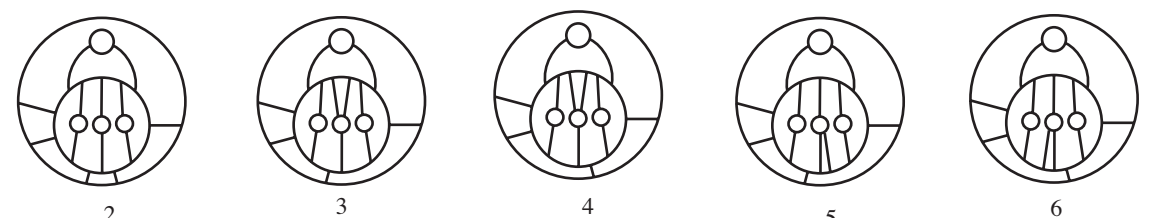

(iid)
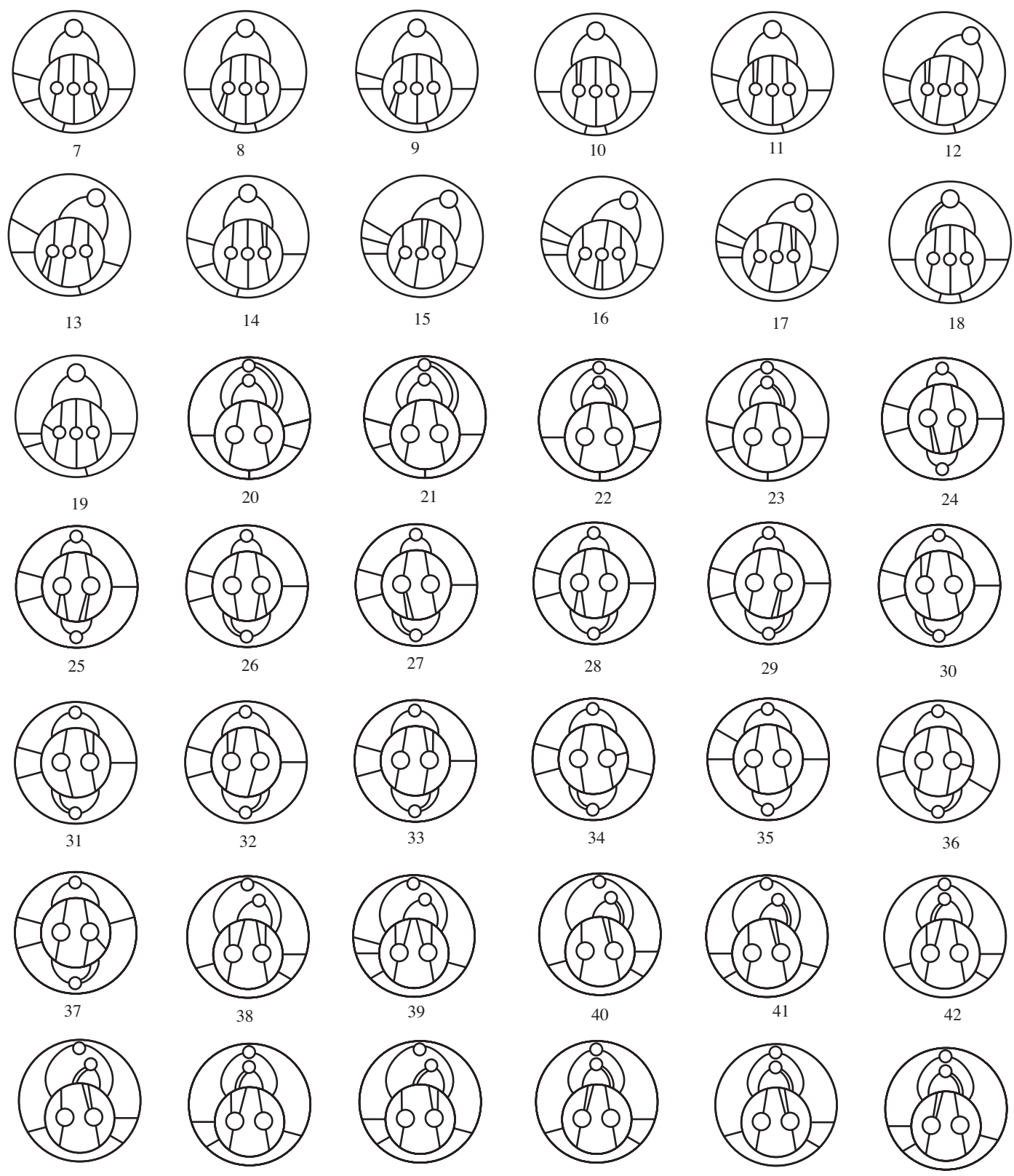

45
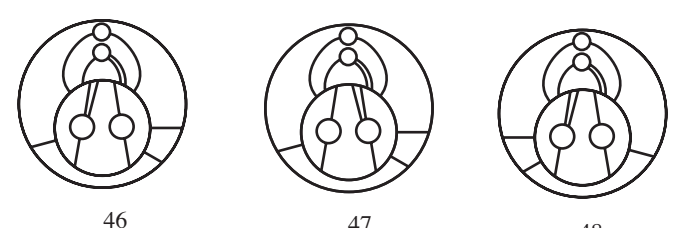

48 
Table 4. DE-graphs with 6 disks
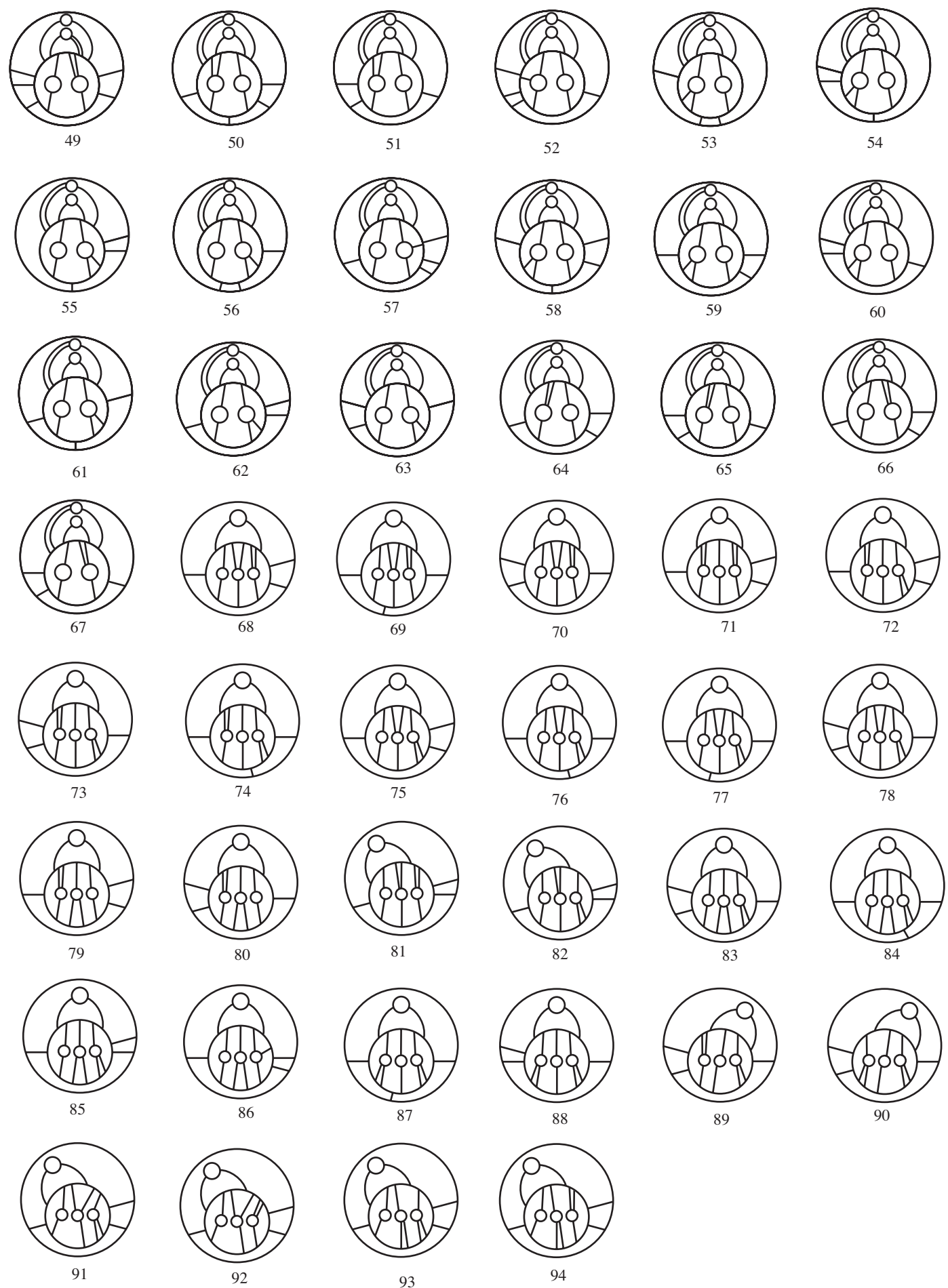